$\xi$

\title{
Dominating Energy of Operations on Intuitionistic Fuzzy Graphs
}

\author{
A.Kalimulla ${ }^{1}$, R.Vijayaragavan ${ }^{2}$, S. Sharief Basha $^{3}$ \\ ${ }^{1}$ Research Scholar, Thiruvalluvar University, Serkadu,Vellore, TN, India \\ ${ }^{2}$ Associate professor, Department of mathematics, Thiruvalluvar University, Serkadu,Vellore, TN,India. \\ ${ }^{3}$ Assistant Professor(Sr),Department of mathematics, S A S, VIT, Vellore, TN, India \\ *Corresponding author E-mail: shariefbasha.s@vit.ac.in
}

\begin{abstract}
The concept of energy of an Intuitionistic Fuzzy Graph is extended to dominating Energy in operations on Intuitionistic Fuzzy Graph. In this paper, We have obtained the value of dominating Energy in different operations such as complement, Union, Join, Cartesian product and composition between two intuitionistic Fuzzy graphs. Also we study the relation between the dominating Energy in the operations on two Intuitionistic Fuzzy Graphs.
\end{abstract}

Keywords: Intuitionistic fuzzy Graph, complements, union, Join of two intuitionistic fuzzy Graph.

\section{Introduction}

Fuzzy set has emerged as a potential area of interdisciplinary research and fuzzy graph theory is of recent interest. The concept of a fuzzy graph relation was defined by Zadeh[18] and it has found applications in the analysis of cluster patterns. Rosen field [14 ] considered the fuzzy relations on fuzzy relations on fuzzy sets and developed the structure of fuzzy graphs.

Atanassov [2,3]introduced the concept of intuitionistic fuzzy relation and intuitionistic fuzzy graphs(IFG).Recent on the theory of intuitionistic fuzzy sets (IFS) has been witnessing an exponential growth of mathematics and its applications. This ranges from normal mathematics to computer sciences, information sciences and communication systems. Graph spectrum appears in problems in Statistical physics and in combinatorial optimization problems in mathematics. Spectrum of a graph also plays an important role in pattern recognition, modelling virus propagation in computer networks and in securing personal data in databases. A concept related to the spectrum of a graph is that of energy.

Let $d_{i}$ be the degree of $i^{\text {th }}$ vertex of $G, i=1,2, \ldots, n$. The spectrum of the graph $\mathrm{G}$, consisting of the numbers $\lambda_{1}, \lambda_{2}, \ldots, \lambda_{n}$ is the spectrum of its adjacency matrix [8].The Laplacian spectrum of the graph $\mathrm{G}$, consisting of the numbers $\mu_{1}, \mu_{2}, \ldots, \mu_{n}$ is the spectrum of its Laplacian matrix.

The ordinary and laplacian graph eigen values obey the following well-known relations

$$
\begin{aligned}
& \sum_{i=1}^{n} \lambda_{i}=0, \quad \sum_{i=1}^{n} \lambda_{i}^{2}=2 m, \quad \sum_{i=1}^{n} \mu_{i}=2 m \\
& \sum_{i=1}^{n} \mu_{i}^{2}=2 m+\sum_{i=1}^{n} d_{i}^{2}
\end{aligned}
$$

In 1960, the study of domination in graphs was begun. In 1862 C.F. De Jaenisch [5] attempted to determine the minimum number of queens required to cover a $n \times n$ chess board. Cockayne [4] introduced the independent domination number in graphs. Domination in graphs has applications to several fields. A. Somasundaram and S. Somasundaram [15 ] introduced domination in fuzzy graphs in terms of effective edges. A. Nagoorgani and V.T. Chandrasekaran [10] introduced domination using strong arcs. R. Parvathi and G. Thamizhendhi [12 ] was introduced dominating set, domination number, independent set, total dominating and total domination number in intuitionistic fuzzy graphs. Study on domination concepts in intuitionistic fuzzy graphs are more convenient that fuzzy graphs, which is useful in the traffic density and telecommunication systems.

This paper is organized as follows. In section 2, we give all the basic definitions related to Laplacian energy of an intuitionistic fuzzy graph and domination in an intuitionistic fuzzy graph. .In section 3, we defined the dominating laplacian energy of different operations of an intuitionistic fuzzy graphs and in section 4 , we give the conclusion

\section{Preliminaries}

\subsection{Intuitionistic Fuzzy Graph}

Definition 2.1.1:-

[11] An intuitionistic fuzzy graph is defined as $\mathrm{G}=(\mathrm{V}, \mathrm{E}, \boldsymbol{\mu}, \boldsymbol{\gamma})$ where $\mathrm{V}$ is the set of vertices and $\mathrm{E}$ is the set of edges,$\mu$ is a fuzzy membership function defined on $\mathrm{VXV}$ and $\gamma$ is a fuzzy non membership function we define $\mu\left(v_{i}, v_{j}\right)$ by $\mu_{i j}$ and $\gamma\left(v_{i}, v_{j}\right)$ by $\gamma_{i j}$ such that (i) $0 \leq \mu_{i j}+\gamma_{i j} \leq 1$ (ii) $0 \leq \mu_{i j}, \gamma_{i j}, \pi_{i j} \leq 1$ where $\pi_{i j}=1-\mu_{i j}-\gamma_{i j}$.Hence $(V \times V, \mu, \gamma)$ is an Intuitionistic fuzzy graph. 


\section{Example 2.1.2:}

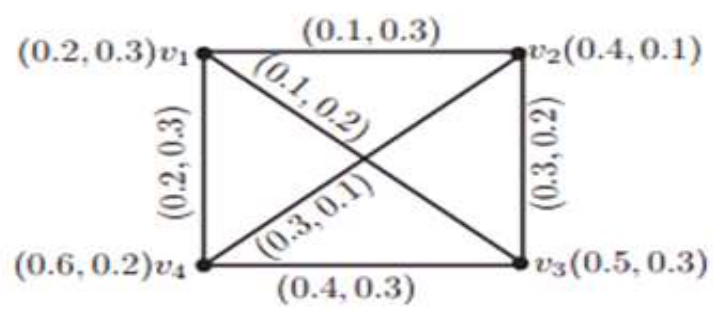

Figure 1 : Intuitionistic Fuzzy Graph $G$.

\section{3: Domination in an Intuitionistic Fuzzy Graph}

Definition 2.3.1:-

An intuitionistic Fuzzy graph is of the form $G=(V, E)$ where (i) $V=\left\{v_{1}, v_{2}, \ldots, v_{n}\right\}$ such that $\mu: V \rightarrow[0,1] \quad \gamma$ : $V \rightarrow[0,1]$ denote the degree of membership and non membership of the element $v \in V$ respectively and $0 \leq \mu_{1}\left(v_{i}\right)+\gamma_{i}\left(v_{i}\right) \leq 1$ for every $v_{i} \in V_{i}(\mathrm{i}=1,2, \ldots, \mathrm{n})$ (ii) $E \subseteq V \times V \quad$ where $\quad \mu_{2}: V \times V \rightarrow[0,1] \quad$ and $\gamma_{2}: V \times V \rightarrow[0,1] \quad$ are such $\mu_{2}\left(v_{i}, v_{j}\right) \leq \mu_{1}\left(v_{i}\right) \wedge \mu_{1}\left(v_{j}\right)$ $\gamma_{2}\left(v_{i}, v_{j}\right) \leq \gamma_{1}\left(v_{i}\right) \wedge \gamma_{1}\left(v_{j}\right)$ and $0 \leq \mu_{2}\left(v_{i}, v_{j}\right)+\gamma_{2}\left(v_{i}, v_{j}\right) \leq 1$

\section{Definition 2.3.2:-}

An $\operatorname{arc}\left(v_{i}, v_{j}\right)$ of an intuitionistic fuzzy graph $\mathrm{G}$ is called a strong arc if $\mu_{2}\left(v_{i}, v_{j}\right) \leq \mu_{1}\left(v_{i}\right) \wedge \mu_{1}\left(v_{j}\right) \quad$ and $\gamma_{2}\left(v_{i}, v_{j}\right) \leq \gamma_{1}\left(v_{i}\right) \wedge \gamma_{1}\left(v_{j}\right)$.

\section{Definition 2.3.3:-}

Let $\mathrm{G}=(\mathrm{V}, \mathrm{E})$ an intuitionistic Fuzzy graph. Let $u, v \in V$,we say that $\mathrm{u}$ dominates $\mathrm{v}$ in $\mathrm{G}$ if there exist a strong are between them. A subset $D \subseteq V$ is said to be dominating set In $\mathrm{G}$ if for every $v \in V-D$, there exist $\mathrm{u}$ in $\mathrm{D}$ such that $\mathrm{u}$ dominates.

\section{Definition 2.3.4:-}

A dominating set $\mathrm{D}$ of an intuitionistic fuzzy is said to be minimal dominating set if no proper subset of $\mathrm{D}$ is a dominating set. Minimum cardinality among all minimal dominating set is called the intuitionistic fuzzy domination number.

\section{4: Dominating Energy in an Intuitionistic Fuzzy Graph}

In this section, we consider an intuitionistic fuzzy graph $G=(V, E, \mu, \gamma)$ then we define $\left(\mu_{1}, \gamma_{1}\right): V \rightarrow[0,1]$ and prove that $\left(\mu_{1}, \gamma_{1}\right)$ is an intuitionistic fuzzy set.
Definition 2.4.1:-

Let $G=\left(V, E, \mu, \gamma, \mu_{1}, \gamma_{1}\right)$ be a dominating fuzzy graph .A dominating intuitionistic fuzzy adjacency matrix $D(G)=\left\lfloor d_{i j}\right\rfloor$ where

$$
d_{i j}=\left\{\begin{array}{lr}
\left(\mu_{i j}, \gamma_{i j}\right) & \text { if }\left(v_{i}, v_{j}\right) \in E \\
(1,1) & \text { if } i=j \text { and } v_{i} \in D \\
(0,0) & \text { otherwise }
\end{array}\right.
$$

This dominating intuitionistic fuzzy adjacency matrix $\mathrm{D}(\mathrm{G})$ can be written as $D(G)=\left(\mu_{D}(G), \gamma_{D}(G)\right)$

where

$$
\mu_{D}(G)=\left\{\begin{array}{lr}
\mu_{i j} & \text { if }\left(v_{i}, v_{j}\right) \in E \\
1 & \text { if } i=j \text { and } v_{i} \in D \\
0 & \text { otherwise }
\end{array}\right.
$$

and

$$
\gamma_{D}(G)=\left\{\begin{array}{lr}
\gamma_{i j} & \text { if }\left(v_{i}, v_{j}\right) \in E \\
1 & \text { if } i=j \text { and } v_{i} \in D \\
0 & \text { otherwise }
\end{array}\right.
$$

Let us illustrate these definitions in the following example In Figure 1 , Consider an IFG, $\mathrm{G}=(\mathrm{V}, \mathrm{E})$ such that $\mathrm{V}=$ $\left\{v_{1}, v_{2}, v_{3}, v_{4}\right\}$

$\mathrm{E}=\left\{\left(v_{1} v_{2}\right),\left(v_{2} v_{3}\right),\left(v_{1} v_{4}\right),\left(v_{3} v_{4}\right),\left(v_{2} v_{4}\right),\left(v_{1} v_{3}\right)\right\}$. Consider a dominating intuitionistic fuzzy graph $G=\left(V, E, \mu, \gamma, \mu_{1}, \gamma_{1}\right)$ where $V=\left\{v_{1}, v_{2}, v_{3}, v_{4}\right\}$

and $\mu_{1}, \gamma_{1}$ are given by $\mu_{1}: V \rightarrow[0,1]$ and $\gamma_{1}: V \rightarrow[0,1]$ where

$\mu_{1}\left(v_{1}\right)=\max _{v_{j}}\left(\mu\left(v_{1}, v_{j}\right)\right)$

$=\max \left(\mu\left(v_{1}, v_{2}\right), \mu\left(v_{1}, v_{4}\right), \mu\left(v_{1}, v_{3}\right)\right)$

$=\max (0.1,0.2,0.1)=0.2$.

Similarly

$\mu_{1}\left(v_{2}\right)=\max (0.1,0.3,0.3)=0.3$

$\mu_{1}\left(v_{3}\right)=\max (0.1,0.4,0.3)=0.4$

$\mu_{1}\left(v_{4}\right)=\max (0.4,0.3,0.2)=0.4$ and

$\gamma_{1}\left(v_{1}\right)=\min _{v_{j}}\left(\gamma\left(v_{i}, v_{j}\right)\right)$

$=\min \left(\gamma\left(v_{1}, v_{2}\right), \gamma\left(v_{1}, v_{4}\right), \gamma\left(v_{1}, v_{3}\right)\right)=\min (0.3,0.3,0.2)=0.2$

Similar-

ly

$\gamma_{1}\left(v_{2}\right)=\min _{v_{j}}\left(\gamma\left(v_{i}, v_{j}\right)\right)=\min \left(\gamma\left(v_{2}, v_{1}\right), \gamma\left(v_{2}, v_{3}\right), \gamma\left(v_{2}, v_{4}\right)\right)=\min (0.3,0.2,0.1)=0.1$

$\gamma_{1}\left(v_{3}\right)=\min _{v_{j}}\left(\gamma\left(v_{i}, v_{j}\right)\right)=\min \left(\gamma\left(v_{3}, v_{1}\right), \gamma\left(v_{3}, v_{2}\right), \gamma\left(v_{3}, v_{4}\right)\right)=\min (0.2,0.2,0.3)=0.2$

$\gamma_{1}\left(v_{4}\right)=\min _{v_{j}}\left(\gamma\left(v_{i}, v_{j}\right)\right)=\min \left(\gamma\left(v_{4}, v_{1}\right), \gamma\left(v_{4}, v_{3}\right), \gamma\left(v_{4}, v_{2}\right)\right)=\min (0.3,0.3,0.1)=0.1$ 
Here $V_{1}$ dominates $V_{3}$ and $V_{2}$ dominates $V_{4}$ because $\mu\left(v_{1}, v_{3}\right) \leq \mu_{1}\left(v_{1}\right) \wedge \mu_{1}\left(v_{3}\right)$

$\gamma\left(v_{1}, v_{3}\right) \leq \gamma_{1}\left(v_{1}\right) \wedge \gamma_{1}\left(v_{3}\right)$

and

$\mu\left(v_{2}, v_{4}\right) \leq \mu_{1}\left(v_{2}\right) \wedge \mu_{1}\left(v_{4}\right)$

$\gamma\left(v_{2}, v_{4}\right) \leq \gamma_{1}\left(v_{2}\right) \wedge \gamma_{1}\left(v_{4}\right)$

Here $V=\left\{v_{1}, v_{2}, v_{3}, v_{4}\right\}$ and Consider a dominating intuitionistic fuzzy graph $G=\left(V, E, \mu, \gamma, \mu_{1}, \gamma_{1}\right)$ where $D=\left\{v_{1}, v_{2}\right\}, V-D=\left\{v_{3}, v_{4}\right\}$

Note that $|D|=2=$ sum of diagonal elements .

For the graph in figure-1,

$$
A_{D}(G)=\left[\begin{array}{cccc}
(1,1) & (0.1,0.3) & (0.1,0.2) & (0.2,0.3) \\
(0.1,0.3) & (1,1) & (0.3,0.2) & (0.3,0.1) \\
(0.1,0.2) & (0.3,0.2) & (0,0) & (0.4,0.3) \\
(0.2,0.3) & (0.3,0.1) & (0.4,0.3) & (0.0)
\end{array}\right]
$$

Where $A_{D}\left(\mu(G)=\left[\begin{array}{cccc}1 & 0.1 & 0.1 & 0.2 \\ 0.1 & 1 & 0.3 & 0.3 \\ 0.1 & 0.3 & 0 & 0.4 \\ 0.2 & 0.3 & 0.4 & 0\end{array}\right]\right.$ and

$A_{D}(\gamma(G))=\left[\begin{array}{cccc}1 & 0.3 & 0.2 & 0.3 \\ 0.3 & 1 & 0.2 & 0.1 \\ 0.2 & 0.2 & 0 & 0.3 \\ 0.3 & 0.1 & 0.3 & 0\end{array}\right]$

Definition 2.4.2:-

The eigen values of dominating intuitionistic fuzzy adjacency matrix $\mathrm{D}(\mathrm{G})$ is defined as $(\mathrm{X}, \mathrm{Y})$ where $\mathrm{X}$ is the set of eigen values of $\mu_{D}(G)$ and $\gamma$ is the set of eigen values of $\gamma_{D}(G)$.The energy of a dominating intuitionistic fuzzy graph $G=\left(V, E, \mu, \gamma, \mu_{1}, \gamma_{1}\right) \quad$ is defined $\left(\sum_{\lambda_{i} \in X}\left|\lambda_{i}\right|, \sum_{\delta_{I} \in Y}\left|\delta_{i}\right|\right)$ Where $\sum_{\lambda_{i} \in X}\left|\lambda_{i}\right|$ is the sum of the absolute values of the eigen values of $\mu_{D}(G)$ and it is denoted by the energy of the membership matrix $E\left(\mu_{D}(G)\right)$ and $\sum_{\delta_{i} \in Y}\left|\delta_{i}\right|$ is the sum of the absolute values of the eigen values of $\gamma_{D}(G)$ and it is denoted by the energy of the membership matrix $E\left(\gamma_{D}(G)\right)$.

\section{Dominating Energy in Operations on Intui- tionistic Fuzzy Graphs}

\subsection{Dominating Energy in Complement of an Intuition- istic Fuzzy Graph}

Definitions 3.1.1: -The complement of an intuitionistic fuzzy Graph $\mathrm{G}=(\mathrm{V}, \mathrm{E})$ is an intuitionistic fuzzy graph, $\overline{\boldsymbol{G}}=\langle\overline{\boldsymbol{V}}, \bar{E}\rangle$ where

$\bar{\mu}_{1 i}=\boldsymbol{\mu}_{1 \mathrm{i}}$ and $\bar{\gamma}_{1 i}=\gamma_{1 i}$, for all $\mathrm{i}=1,2, \ldots, \mathrm{n}$ $\bar{\mu}_{2 i j}=\quad \mu_{1 \mathrm{i}} \quad \mathrm{ij}_{\mathrm{j}} \quad-\mu_{2 \mathrm{ij}} \quad$ and $\vec{\gamma}_{2 i j}=\gamma_{1 i} \cdot \gamma_{1 j}-\gamma_{2 i j}$ for all $i, j=1,2, \ldots, n$.

Example 3.1.2:-

Let $\mathrm{V}=\left\{v_{1}, v_{2}, v_{3}, v_{4}\right\}$

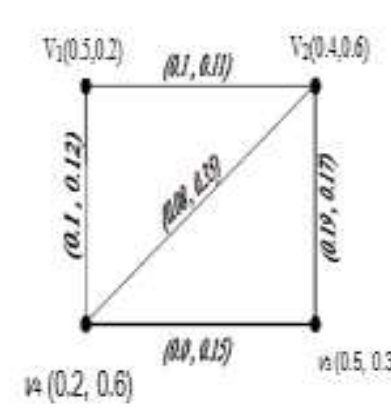

FIG 2(a)

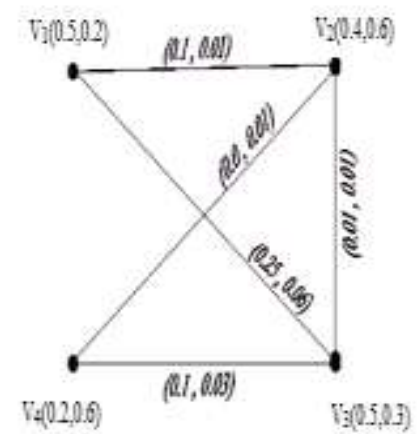

FIG 2 (b)
First we find the dominating Energy of Intuitionistic fuzzy Graph $\mathrm{G}(\mathrm{V}, \mathrm{E})$ :-

Consider a dominating intuitionistic fuzzy GraphG=(V,E, $\boldsymbol{\mu}$, $\left.\gamma, \mu_{1}, \gamma_{1}\right)$ where $\mathrm{v}=\left\{\mathrm{v}_{1}, \mathrm{v}_{2}, \mathrm{v}_{3}, \mathrm{v}_{4}\right\}$

and

$\gamma_{\text {mil }}$ are given bv $\mu_{1}: V \rightarrow[0,1]$ and $\gamma_{1}: V \rightarrow[0,1]$ where

$\mu_{1}\left(v_{1}\right)=\max \left[\mu\left(v_{1} v_{2}\right), \mu\left(v_{1} v_{4}\right)\right]=\max [0.1,0.1]=0.1$.

$\mu_{1}\left(v_{2}\right)=\max \left[\mu\left(v_{2} v_{1}\right), \mu\left(v_{2}, v_{3}\right)\right]=\max [0,1,0.19]=0.19$.

$\mu_{1}\left(v_{3}\right)=\max \left[\mu\left(v_{3} v_{2}\right), \mu\left(v_{3}, v_{4}\right)\right]=\max [0,19,0,0]=0.19$.

$\mu_{1}\left(v_{4}\right)=\max \left[\mu\left(v_{4} v_{3}\right) \mu\left(v_{4} v_{1}\right)\right]=\max [0.0,0.1]=0.1$.

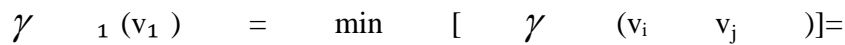
$\min \left[\gamma\left(\mathrm{v}_{1} \mathrm{v}_{2}\right), \gamma\left(\mathrm{v}_{1} \mathrm{v}_{4}\right)\right]=\min [0,11,0,12]=0.11$.

$\gamma_{1}\left(\mathrm{v}_{2}\right)=\overline{\min }\left[\gamma\left(\mathrm{v}_{2} \mathrm{v}_{1}\right),{ }^{-} \gamma\left(\mathrm{v}_{2} \mathrm{v}_{3}\right)\right]=\min [0.11,0.17]=0.11$.

$\gamma_{1}\left(\mathrm{v}_{3}\right)=\min \left[\gamma\left(\mathrm{v}_{3} \mathrm{v}_{2}\right), \gamma\left(\mathrm{v}_{3} \mathrm{v}_{4}\right)\right]=\min [0.17,0.15]=0.15$.

$\gamma_{1}\left(v_{4}\right)=\min \left[\gamma\left(v_{4} v_{3}\right), \gamma\left(v_{4} v_{1}\right)\right]=\min [0.15,0.12]=0.12$.

Here $\mathrm{v}_{1}$ dominates $\mathrm{v}_{2}$ because

$\mu\left(v_{1} v_{2}\right) \leq \mu_{1}\left(v_{1}\right) \wedge \mu_{1}\left(v_{2}\right)$

$0.1 \leq 0.1 \wedge 0.19$

$\gamma\left(v_{1} v_{2}\right) \leq \gamma_{1}\left(v_{1}\right) \wedge \gamma_{1}\left(v_{2}\right)$

$0.11 \leq 0.11 \wedge 0.11$

Here $v_{3}$ dominates $v_{4}$ because

$\mu\left(v_{3} v_{4}\right) \leq \mu_{1}\left(v_{3}\right) \wedge \mu_{1}\left(v_{4}\right)$

$0.0 \leq 0.19 \wedge 0.1$

$$
\bar{V}=V \quad \begin{array}{ll}
\gamma\left(v_{3} v_{4}\right) \leq \gamma_{1}\left(v_{3}\right) \wedge \gamma_{1}\left(v_{4}\right) \\
0.15 \leq 0.15 \wedge 0.12
\end{array}
$$

Here $\mathrm{V}=\left\{\mathrm{v}_{1}, \mathrm{v}_{2}, \mathrm{v}_{3}, \mathrm{v}_{4}\right\}$ and $\mathrm{D}=\left\{\mathrm{v}_{1}, \mathrm{v}_{3}\right\}, \mathrm{V}-\mathrm{D}=\left\{\mathrm{v}_{2}, \mathrm{v}_{4}\right\}|\mathrm{D}|$ $=2=$ sum of dominating elements. 


\section{Definition :}

Let $\mathrm{G}=\left[\mathrm{V}, \mathrm{E}, \mu, \gamma, \mu_{1}, \gamma_{1}\right]$ be a dominating Intuitionistic Fuzzy Graph. A dominating Intuitionistic Fuzzy adjacency matrix $\mathrm{D}(\mathrm{G})=\left(\mathrm{d}_{\mathrm{j}} \mathrm{j}\right)$ where

$d_{i j}=\left\{\begin{array}{cc}\left(\mu_{i j}, \gamma_{i j}\right) & \text { if }\left(v_{i}, v_{j}\right) \in E \\ (1,1) & \text { if } i=j \& v_{i} \in D \\ 0 & \text { otherwise }\end{array}\right.$

$D(G)=\left[\begin{array}{cccc}(1,1) & (0.1,0.11) & (0,0) & (0.1,0.12) \\ (0.1,0.11) & (0,0) & (0.19,0.17) & (0,0) \\ (0,0) & (0.19,0.17) & (1,1) & (0.0,0.15) \\ (0.1,0.12) & (0,0) & (0.0,0.15) & (0,0)\end{array}\right]$

$\mu_{D}(G)=\left[\begin{array}{cccc}1 & 0.1 & 0 & 0.1 \\ 0.1 & 0 & 0.19 & 0 \\ 0 & 0.19 & 1 & 0 \\ 0.1 & 0 & 0 & 0\end{array}\right]$

$\gamma_{D}(G)=\left[\begin{array}{cccc}1 & 0.11 & 0 & 0.12 \\ 0.11 & 0 & 0.17 & 0 \\ 0 & 0.17 & 1 & 0.15 \\ 0.12 & 0 & 0.15 & 0\end{array}\right]$

Eigen values of

$\mu_{D}(G)=\{-0.0465,-0.0074,1,0074,1,0465\}=$ Spectrum of $\mu_{D}(G)$

Eigen values of $\gamma_{D}(G)=\{-0.0725,-0.0002,1.0002,1.0725\}=$

Spectrum of $\gamma_{D}(G)$

Dominating Energy of Intuitionistic fuzzy graph $\mathrm{G}=(\mathrm{V}, \mathrm{E})=$

$\left[\sum_{\lambda_{i} \in X}\left|\lambda_{i}\right|, \sum_{\delta_{i} \in Y}\left|\delta_{i}\right|\right]=[2.1078,2.1454]$.

Now we find the Dominating Energy of complement of an Intuitionistic Fuzzy Graph G(V,E):-

Consider a dominating Intuitionistic Fuzzy graph

$\left.\mathrm{G}=\mathrm{V}, \mathrm{E}, \mu, \gamma, \mu_{1}, \gamma_{1}\right) \quad$ where $\mathrm{V}=\left\{\mathrm{v}_{1}, \mathrm{v}_{2}, \mathrm{v}_{3}, \mathrm{v}_{4}\right\}$ and $\mu_{1}$ and $\gamma_{1}$ given by $\mu_{1}: \mathrm{V} \rightarrow[0.1]$ and $\gamma_{1}: \mathrm{V} \rightarrow[0,1]$ where

$$
\begin{aligned}
\mu_{1}\left(v_{1}\right) & =\max \left[\mu\left(v_{1} v_{2}\right), \mu\left(v_{1} v_{3}\right)\right] \\
& =\max [0.1,0.25]=0.25
\end{aligned}
$$

$\mu_{1}\left(\mathrm{v}_{2}\right)=\max \left[\mu\left(\begin{array}{ll}\mathrm{v}_{2} & \mathrm{v}_{1}\end{array}\right), \mu\left(\mathrm{v}_{2} \mathrm{v}_{4}\right), \mu\left(\mathrm{v}_{2} \mathrm{v}_{3}\right)\right]=\max [0.1,0.0,0.01]=0$. 1

$\mu_{1}\left(\mathrm{v}_{3}\right)=\max \left[\mu\left(\mathrm{v}_{3} \mathrm{v}_{4}\right), \mu\left(\mathrm{v}_{3} \mathrm{v}_{1}\right), \mu\left(\mathrm{v}_{3} \mathrm{v}_{2}\right)\right]=\max [0.1,0.25,0.01]=$ 0.25

$\mu_{1}\left(\mathrm{v}_{4}\right)=\max \left[\mu\left(\mathrm{v}_{4} \mathrm{v}_{3}\right)\right], \mu\left(\mathrm{v}_{4} \mathrm{v}_{2}\right),=\max [0.1,0.0]=0.1$

$\gamma \quad{ }_{1}\left(\mathrm{v}_{1}\right)=\min [\quad \gamma$

$\mathrm{vj})]=\min \left[\gamma\left(\mathrm{v}_{1} \mathrm{v}_{2}\right), \gamma\left(\mathrm{v}_{1} \mathrm{v}_{3}\right)\right]=\min [0.01,0.06]=0.01$

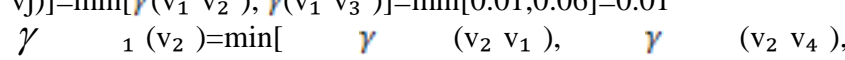

$\left.\gamma\left(\mathrm{v}_{2} \mathrm{v}_{3}\right)\right]=\min [0.01,0.01,0.01]=0.01$

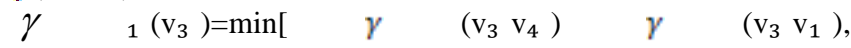

$\left.\gamma\left(\mathrm{v}_{3} \mathrm{v}_{2}\right)\right]=\min [0.03,0.06,0.01]=0.01$

$\gamma_{1}\left(\mathrm{v}_{4}\right)=\min \left[\gamma\left(\mathrm{v}_{4} \mathrm{v}_{3}\right), \gamma\left(\mathrm{v}_{4} \quad \mathrm{v}_{2}\right)\right]=\min [0.03,0.01]=0.01$

Here $v_{1}$ dominates $v_{2}$ because $\mu\left(v_{1} v_{2}\right) \leq \mu_{1}\left(v_{1}\right) \wedge \mu_{1}\left(v_{2}\right)$

$0.1 \leq 0.25 \wedge 0.1$

$\gamma\left(v_{1} v_{2}\right) \leq \gamma_{1}\left(v_{1}\right) \wedge \gamma_{1}\left(v_{2}\right)$

$0.01 \leq 0.01 \wedge 0.01$

Here $v_{2}$ dominates $v_{3}$ because

$\mu\left(v_{2} v_{3}\right) \leq \mu_{1}\left(v_{2}\right) \wedge \mu_{1}\left(v_{3}\right)$

$0.01 \leq 0.1 \wedge 0.25$

$\gamma\left(v_{2} v_{3}\right) \leq \gamma_{1}\left(v_{2}\right) \wedge \gamma_{1}\left(v_{3}\right)$

$0.01 \leq 0.01 \wedge 0.01$

Here $\mathrm{V}=\left\{\mathrm{v}_{1}, \mathrm{v}_{2}, \mathrm{v}_{3}, \mathrm{v}_{4}\right\}$ and $\mathrm{D}=\left\{\mathrm{v}_{1}, \mathrm{v}_{2}\right\} \quad \mathrm{V}-\mathrm{D}=\left\{\mathrm{v}_{3}, \mathrm{v}_{4}\right\}$

$|\mathrm{D}|=2=$ sum of dominating elements

$D(G)=\left[\begin{array}{cccc}(1,1) & (0.1,0.01) & (0.25,0.06) & (0,0) \\ (0.1,0.01) & (1,1) & (0.01,0.01) & (0,0.01) \\ (0.25,0.06) & (0.01,0.01) & (0,0) & (0.1,0.03) \\ (0,0) & (0,0.01) & (0.1,0.03) & (0,0)\end{array}\right]$

$\mu_{D}(G)=\left[\begin{array}{cccc}1 & 0.1 & 0.25 & 0 \\ 0.1 & 1 & 0.01 & 0 \\ 0.25 & 0.01 & 0 & 0.1 \\ 0 & 0 & 0.1 & 0\end{array}\right]$,

$\gamma_{D}(G)=\left[\begin{array}{cccc}1 & 0.01 & 0.06 & 0 \\ 0.01 & 1 & 0.01 & 0.01 \\ 0.06 & 0.01 & 0 & 0.03 \\ 0 & 0.01 & 0.03 & 0\end{array}\right]$

Eigen values of $\mu_{\mathrm{D}}(\mathrm{G})=\{-0.1314,0.0717,0.9260,1.1337\}=2.2628$

Eigen values of $\gamma_{D}(\mathrm{G})=\{-0.0318,0.0280,0.9912,1.0126\}=2.0636$

Dominating Energy of Complement of Intuitionistic Fuzzy graph $\mathrm{G}=(\mathrm{V}, \mathrm{E})$

$=\left[\sum_{\lambda_{i} \in X}\left|\lambda_{i}\right|, \sum_{\delta_{i} \in Y}\left|\delta_{i}\right|\right]=[2.2628,2.0636]$.

Comparison between Dominating Energy of Intuitionist Fuzzy Graph and its complement:- we observe here that laplacian energy of membership function of an intuitionistic fuzzy graph is less than its complement and laplacian energy of non membership function of an intuitionistic fuzzy graph is greater than its complement.

\subsection{Dominating Energy in Union of Intuitionistic Fuzzy Graph}

Definition 3.2.1:-

Let $\mathrm{G}_{1}=\left\langle\mathrm{V}_{1}, \mathrm{E}_{1}\right\rangle$ and $\mathrm{G}_{2}=\left\langle\mathrm{V}_{2}, \mathrm{E}_{2}\right\rangle$ be two Intuitionistic Fuzzy Graphs with $V_{1} \cap V_{2}=\phi$ and $G=G_{1} \cup G_{2}=\left\langle V_{1} \cup V_{2}, E_{1} \cup E_{2}\right\rangle$ be the Union of $G_{1}$ and $G_{2}$. Then the Union of Intuitionistic fuzzy graphs

( $v_{i} \quad G_{1}$ and $G_{2}$ is an intuitionistic fuzzy Graph defined by

$\left(\mu_{1} \cup \mu_{1}^{\prime}\right)(v)= \begin{cases}\mu_{1}(v) & \text { if } v \in v_{1}-v_{2} \\ \mu_{1}^{\prime}(v) & \text { if } v \in v_{2}-v_{1}\end{cases}$

$\left(\gamma_{1} \cup \gamma_{1}^{\prime}\right)(v)=\left\{\begin{array}{ll}\gamma_{1}(v) & \text { if } v \in v_{1}-v_{2} \\ \gamma_{1}^{\prime}(v) & \text { if } v \in v_{2}-v_{1}\end{array}\right.$ and 
$\left(\mu_{2} \cup \mu_{2}^{\prime}\right)\left(v_{i}, v_{j}\right)= \begin{cases}\mu_{2}\left(e_{i j}\right) & \text { if } e_{i j} \in E_{1}-E_{2} \\ \mu_{2}^{\prime}\left(e_{i j}\right) & \text { if } e_{i j} \in E_{2}-E_{1}\end{cases}$

Where $\left(\mu_{1}, \gamma_{1}\right)$ and $\left(\mu_{1}^{\prime}, \gamma_{1}^{\prime}\right)$ refer the vertex membership and non-membership of $\mathrm{G}_{1}$ and $\mathrm{G}_{2}$ respectively; $\left(\mu_{2}, \gamma_{2}\right)$ and $\left(\mu_{2}^{\prime}, \gamma_{2}^{\prime}\right)$ refer the edge membership and non-membership of $\mathrm{G}_{1}$ and $\mathrm{G}_{2}$ respectively.

\section{Example 3.2.2:-}

Let $\mathrm{V}_{1}=\left\{\mathrm{v}_{2}, \mathrm{v}_{3}, \mathrm{v}_{4}\right\}$ and $\mathrm{V}_{2}=\left\{\mu_{1}, \mu_{2}\right\}$ such that $v_{1} \cap v_{2}=\phi$

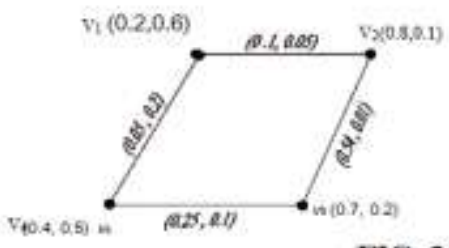

FIG 3(a): $G_{1} \& G_{2}$

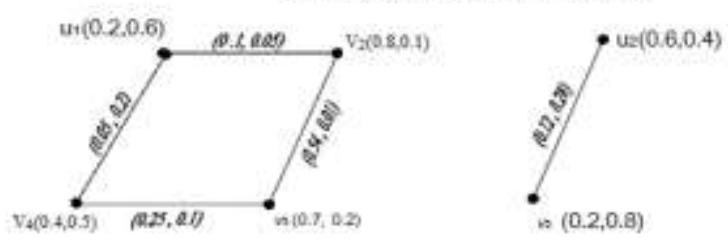

FIG 3(b) : $\mathrm{G}_{1} \mathrm{UG}_{2}$

First we find the dominating Energy of Intuitionistic Fuzzy Graph $\mathrm{G}_{1}(\mathrm{~V}, \mathrm{E})$ :-

Consider a dominating intuitionistic fuzzy Graph

$\mathrm{G}_{1}=\left(\mathrm{V}, \mathrm{E}, \boldsymbol{\mu}, \Upsilon, \mu_{1}, \Upsilon_{1}\right)$ where $\mathrm{v}=\left\{\mathrm{v}_{1}, \mathrm{v}_{2}, \mathrm{v}_{3}, \mathrm{v}_{4}\right\}$ and $\mu_{1}$ and

$\gamma_{1}$ are given by $\mu_{1} \mathrm{aV} \rightarrow[0,1]$ and $\gamma_{1}: V \rightarrow[0,1]$ where

$$
\begin{aligned}
\mu_{1}\left(v_{1}\right) & =\max \left[\mu\left(v_{1} v_{2}\right), \mu\left(v_{1} v_{4}\right)\right] \\
& =\max [0.1,0.05]=0.1 \\
\mu_{1}\left(v_{2}\right) & =\max \left[\mu\left(v_{2} v_{1}\right), \mu\left(v_{2} v_{3}\right)\right] \\
& =\max [0.1,0.54]=0.54 . \\
\mu_{1}\left(v_{3}\right) & =\max \left[\mu\left(v_{3} v_{2}\right), \mu\left(v_{3} v_{4}\right)\right] \\
& =\max [0.54,0.25]=0.54 .
\end{aligned}
$$

Here $v_{2}$ dominates $v_{3}$ because

$\mu\left(v_{2} v_{3}\right) \leq \mu_{1}\left(v_{2}\right) \wedge \mu_{1}\left(v_{3}\right)$

$0.54 \leq 0.54 \wedge 0.54$

$\gamma\left(v_{2} v_{3}\right) \leq \gamma_{1}\left(v_{2}\right) \wedge \gamma_{1}\left(v_{3}\right)$

$0.01 \leq 0.01 \wedge 0.01$

Here $v_{3}$ dominates $v_{4}$ because

$\mu\left(v_{3} v_{4}\right) \leq \mu_{1}\left(v_{3}\right) \wedge \mu_{1}\left(v_{4}\right)$

$0.25 \leq 0.54 \wedge 0.25$

$\gamma\left(v_{3} v_{4}\right) \leq \gamma_{1}\left(v_{3}\right) \wedge \gamma_{1}\left(v_{4}\right)$

$0.1 \leq 0.01 \wedge 0.1$

Here $\mathrm{V}=\left\{\mathrm{v}_{1}, \mathrm{v}_{2}, \mathrm{v}_{3}, \mathrm{v}_{4}\right\}$ and $\mathrm{D}=\left\{\mathrm{v}_{1}, \mathrm{v}_{2}, \mathrm{v}_{3}\right\} \quad \mathrm{V}-\mathrm{D}=\left\{\mathrm{v}_{4}\right\}$ $|\mathrm{D}|=3=$ sum of dominating elements.

$D\left(G_{1}\right)=\left[\begin{array}{cccc}(1,1) & (0.1,0.05) & (0,0) & (0.05,0.2) \\ (0.1,0.05) & (1,1) & (0.54,0.01) & (0,0) \\ (0,0) & (0.54,0.01) & (1,1) & (0.25,0.1) \\ (0.05,0.2) & (0,0) & (0.25,0.1) & (0,0)\end{array}\right]$

$\mu_{D}\left(G_{1}\right)=\left[\begin{array}{cccc}1 & 0.1 & 0 & 0.05 \\ 0.1 & 1 & 0.54 & 0 \\ 0 & 0.54 & 1 & 0.25 \\ 0.05 & 0 & 0.25 & 0\end{array}\right]$

$\gamma_{D}\left(G_{1}\right)=\left[\begin{array}{cccc}1 & 0.05 & 0 & 0.2 \\ 0.05 & 1 & 0.01 & 0 \\ 0 & 0.01 & 1 & 0.1 \\ 0.2 & 0 & 0.1 & 0\end{array}\right]$

Eigen Values of $\mu_{\mathrm{p}}\left(\mathrm{G}_{1}\right)=[-0.0810,0.5108,1.0000,1.5703]=3.1621$. Eigen values of $\gamma \quad{ }_{\mathrm{D}}\left(\mathrm{G}_{1}\right)=[-0.0478,0.9663,1.0033,1.0782]$ $=3.0956$.

Dominating Energy of UNION of IFG $\bar{G}_{1}=(\bar{V}, \bar{E})$ is $=$

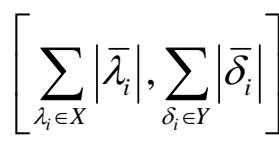

$=(3.1621,3.0956)$

Also we find the dominating Energy of Intuitionistic Fuzzy Graph $\mathrm{G}_{2}(\mathrm{~V}, \mathrm{E})$ :-

Let $V=\left\{v_{1}, v_{2}\right\}$

$\mu_{1}\left(v_{1}\right)=\max \left[\mu\left(v_{1} v_{2}\right)\right]=\max [0.12]=0.12$.

$\mu_{1}\left(v_{4}\right)=\max \left[\mu\left(v_{4} v_{3}\right), \mu\left(v_{4} v_{1}\right)\right]=\max [0.25,0.05]=0.25_{1}\left(v_{2}\right)=\max \left[\mu\left(v_{2} v_{1}\right)\right]=\max [0.12]=0.12$.

$\gamma_{1}\left(\mathrm{v}_{1}\right)=\min \left[\gamma\left(\mathrm{v}_{\mathrm{i}} \mathrm{v}_{\mathrm{j}}\right)\right]=$

$\gamma_{1}\left(\mathrm{v}_{1}\right)=\min \left[\gamma_{\left.\left(\mathrm{v}_{1} \mathrm{v}_{2}\right)\right]}=\min [0.28]=0.28\right.$

$\gamma_{1}\left(v_{2}\right)=\min \left[\gamma_{\left(v_{2} v_{1}\right)}\right]=\min [0.28]=0.28$

Here $v_{1}$ dominates $v_{2}$ because

$\mu\left(v_{1} v_{2}\right) \leq \mu_{1}\left(v_{1}\right) \wedge \mu_{1}\left(v_{2}\right)$

$0.12 \leq 0.12 \wedge 0.12$

$\gamma\left(v_{1} v_{2}\right) \leq \gamma_{1}\left(v_{1}\right) \wedge \gamma_{1}\left(v_{2}\right)$

$0.28 \leq 0.28 \wedge 0.28$

Here $\mathrm{V}=\left\{\mathrm{v}_{1}, \mathrm{v}_{2}\right\}$ and $\mathrm{D}=\left\{\mathrm{v}_{1}\right\}, V-D=\left\{V_{2}\right\}$

$\mathrm{D} \mid=1=$ sum of dominating elements.

$0.1 \leq 0.1 \wedge 0.54$

$\gamma\left(v_{1} v_{2}\right) \leq \gamma_{1}\left(v_{1}\right) \wedge \gamma_{1}\left(v_{2}\right)$

$0.05 \leq 0.05 \wedge 0.01$ 


$$
\begin{aligned}
& D\left(G_{2}\right)=\left[\begin{array}{cc}
(1,1) & (0.12,0.28) \\
(0.12,0.28) & (0,0)
\end{array}\right] \\
& \mu_{D}\left(G_{2}\right)=\left[\begin{array}{cc}
1 & 0.12 \\
0.12 & 0
\end{array}\right] \\
& \gamma_{D}\left(G_{2}\right)=\left[\begin{array}{cc}
1 & 0.28 \\
0.28 & 0
\end{array}\right]
\end{aligned}
$$

Eigen values of $\mu_{\mathrm{D}}\left(\mathrm{G}_{2}\right)=[-0.0142,1.0142]=1.0284$.

Eigen values of $\gamma_{\mathrm{D}}\left(\mathrm{G}_{2}\right)=[-0.0731,1.0731]=1.1462$.

Dominating laplacian energy of Intuitionistic fuzzy graph $\mathrm{G}_{2}$ i.e

$$
\bar{G}_{2}=(\bar{V}, \bar{E}) \text { is }=\left[\sum_{\lambda_{i} \in X}\left|\bar{\lambda}_{i}\right|, \sum_{\delta_{i} \in Y}\left|\bar{\delta}_{i}\right|\right]
$$

$=(1.0284,1.1462)$

Now we find the dominating energy of union of two Intuitionistic fuzzy graphs $G_{1} \cup G_{2}$

$$
\begin{aligned}
\mu_{1}\left(v_{1}\right) & =\max \left[\mu\left(v_{1} v_{2}\right), \mu\left(v_{1} v_{4}\right)\right] \\
& =\max [0.1,0.05]=0.1 \\
\mu_{1}\left(v_{2}\right) & =\max \left[\mu\left(v_{2} v_{1}\right), \mu\left(v_{2} v_{3}\right)\right] \\
& =\max [0.1,0.54]=0.54 \\
\mu_{1}\left(v_{3}\right) & =\max \left[\mu\left(v_{3} v_{2}\right), \mu\left(v_{3} v_{4}\right)\right] \\
& =\max [0.54,0.25]=0.54
\end{aligned}
$$$$
\mu_{1}\left(v_{4}\right)=\max \left[\mu\left(v_{4} v_{3}\right), \mu\left(v_{4} v_{1}\right)\right]=\max [0.25,0.05]=0.25
$$$$
\mu_{1}\left(u_{2}\right)=\max \left[\mu\left(u_{2} u_{1}\right)\right]=\max [0.12]=0.12
$$$$
\gamma_{1}\left(v_{1}\right)=\min \left[\gamma\left(v_{1} v_{2}\right), \gamma\left(v_{1} v_{4}\right)\right]=\min [0.05,0.2]=0.05
$$$$
\gamma_{D}\left(G_{1} \cup G_{2}\right)=\left[\begin{array}{llllll}
1 & 0.05 & 0 & 0.2 & 0 & 0 \\
0.05 & 1 & 0.01 & 0 & 0 & 0 \\
0 & 0.01 & 1 & 0.1 & 0 & 0 \\
0.2 & 0 & 0.1 & 0 & 0 & 0 \\
0 & 0 & 0 & 0 & 1 & 0.28
\end{array}\right]
$$$$
\mu\left(v_{4} v_{1}\right) \leq \mu_{1}\left(v_{4}\right) \wedge \mu_{1}\left(v_{1}\right)
$$$$
\gamma\left(v_{4} v_{1}\right) \leq \gamma_{1}\left(v_{4}\right) \wedge \gamma_{1}\left(v_{1}\right)
$$$$
0.05 \leq 0.25 \wedge 0.1
$$$$
0.2 \leq 0.1 \wedge 0.05
$$

Here $\mathrm{u}_{1}$ dominates $\mathrm{u}_{2}$ because

$$
\begin{aligned}
& \mu_{1}\left(u_{1} u_{2}\right) \leq \mu_{1}\left(u_{1}\right) \wedge \mu_{1}\left(u_{2}\right) \\
& \gamma\left(u_{1} u_{2}\right) \leq \gamma_{1}\left(u_{1}\right) \wedge \gamma_{1}\left(u_{2}\right) \\
& 0.12 \leq 0.12 \wedge 0.12 \\
& 0.28 \leq 0.28 \wedge 0.28
\end{aligned}
$$

Here $\mathrm{V}=\left\{\mathrm{v}_{1}, \mathrm{v}_{2}, \mathrm{v}_{3}, \mathrm{v}_{4}, \mathrm{u}_{1}, \mathrm{u}_{2}\right\}$ and $\mathrm{D}=\left\{\mathrm{v}_{1}, \mathrm{v}_{2}, \mathrm{v}_{3}, \mathrm{u}_{1}\right\} \quad \mathrm{V}-\mathrm{D}=\left\{\mathrm{v}_{4}, \mathrm{u}_{2}\right\}$ $|D|=4=$ Sum of dominating elements.

$D\left(G_{1} \cup G_{2}\right)=\left[\begin{array}{llllll}(1,1) & (0.1,0.05) & (0,0) & (0.05,0.2) & (0,0) & (0,0) \\ (0.1,0.05) & (1,1) & (0.54,0.01) & (0,0) & (0,0) & (0,0) \\ (0,0) & (0.54,0.01) & (1,1) & (0.25,0.1) & (0,0) & (0,0) \\ (0.05,0.2) & (0,0) & (0.25,0.1) & (0,0) & (0,0) & (0,0) \\ (0,0) & (0,0) & (0,0) & (0,0) & (1,1) & (0.12,0.28) \\ (0,0) & (0,0) & (0,0) & (0,0) & (0.12,0.28) & (0,0)\end{array}\right]$

$$
\mu_{D}\left(G_{1} \cup G_{2}\right)=\left[\begin{array}{rrrrrr}
1 & 0.1 & 0 & 0.05 & 0 & 0 \\
0.1 & 1 & 0.54 & 0 & 0 & 0 \\
0 & 0.54 & 1 & 0.25 & 0 & 0 \\
0.05 & 0 & 0.25 & 0 & 0 & 0 \\
0 & 0 & 0 & 0 & 1 & 0.12 \\
0 & 0 & 0 & 0 & 0.12 & 0
\end{array}\right]
$$

$\mu_{1}\left(u_{1}\right)=\max \left[\mu\left(u_{1} u_{2}\right)\right]=\max [0.12]=0.12$.

$\gamma_{1}\left(v_{2}\right)=\min _{\text {Eigen values of }}\left[\gamma\left(v_{2} v_{1}\right), \gamma\left(v_{2} v_{3}\right)\right]=\min [0.85,0.029=0.01$

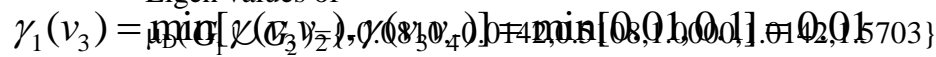

$$
\gamma_{1}\left(u_{1}\right)=\min \left[\gamma\left(u_{1} u_{2}\right)\right]=\min [0.28]=0.28
$$

$\gamma_{1}\left(u_{2}\right)=\min \left[\gamma\left(u_{2} u_{1}\right)\right]=\min [0.28]=0.28$

Here $v_{1}$ dominates $v_{2}$ because

$\mu\left(v_{1} v_{2}\right) \leq \mu_{1}\left(v_{1}\right) \wedge \mu_{1}\left(v_{2}\right)$

$0.1 \leq 0.1 \wedge 0.54$

Here $v_{2}$ dominates $v_{3}$ because

$\mu\left(v_{2} v_{3}\right) \leq \mu_{1}\left(v_{2}\right) \wedge \mu_{1}\left(v_{3}\right)$

$\gamma\left(v_{2} v_{3}\right) \leq \gamma_{1}\left(v_{2}\right) \wedge \gamma_{1}\left(v_{3}\right)$

$0.54 \leq 0.54 \wedge 0.54$

$0.01 \leq 0.01 \wedge 0.01$

Here $v_{3}$ dominates $v_{4}$ because

$\mu\left(v_{3} v_{4}\right) \leq \mu_{1}\left(v_{3}\right) \wedge \mu_{1}\left(v_{4}\right)$

$\gamma\left(v_{3} v_{4}\right) \leq \gamma_{1}\left(v_{3}\right) \wedge \gamma_{1}\left(v_{4}\right)$

$0.25 \leq 0.54 \wedge 0.25$

$0.1 \leq 0.01 \wedge 0.1$

Here $\mathrm{v}_{4}$ not dominates $\mathrm{v}_{1}$ because

$$
=4.1905 \text {. }
$$

\subsection{Dominating Energy in Join of Intutionistic Fuzzy Graphs:-}

Definations 3.3.1:-

The join of two Intutionistic fuzzy graph $\mathrm{G}=\mathrm{G}_{1}+\mathrm{G}_{2}=\left\langle\mathrm{v}_{1} \quad U\right.$ $\mathrm{v}_{2}, \mathrm{E}_{1} \cup \mathrm{E}_{2} \cup \mathrm{E}^{\mathrm{l}}>$ defined by

$\left(\mu_{1}+\mu_{1}^{\prime}\right)(v)=\left(\mu_{1} \cup \mu_{1}^{\prime}\right)(v)$ if $v \in V_{1} \cup V_{2}$

$\left(\gamma+\gamma_{1}{ }^{1}\right)(\mathrm{v})=\left(\gamma_{1} \cup \gamma_{1}{ }^{1}\right)(\mathrm{v})$ If $\mathrm{VE} \mathrm{v}_{1} \cup \mathrm{v}_{2}$ $\left(\mu_{2}+\mu_{2}{ }^{1}\right)\left(\mathrm{v}_{\mathrm{i}} \mathrm{v}_{\mathrm{J}}\right)=\left(\mu_{1} \cup \mu_{2}{ }^{1}\right)\left(\mathrm{v}_{\mathrm{i}} \mathrm{v}_{\mathrm{J}}\right) \quad$ If $\mathrm{v}_{\mathrm{i}} \mathrm{v}_{\mathrm{J}} \in \mathrm{E}_{1} \cup$ $\mathrm{E}_{2}$

$=\left(\mu\left(\mathrm{v}_{\mathrm{i}}\right) \mu_{1}{ }^{1}\left(\mathrm{v}_{\mathrm{J}}\right) \quad\right.$ If $\mathrm{v}_{\mathrm{i}} \mathrm{v}_{\mathrm{J}} \in \mathrm{E}_{1}$

\section{Example 3.3.2:-}

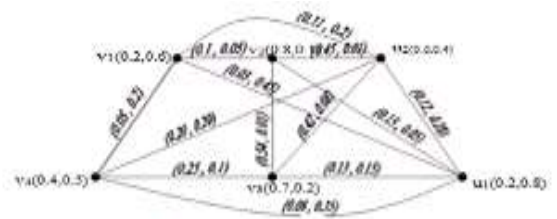

FIC 4: $G-G_{1}+G_{2}$ 
Now we find the dominating Energy of join of IFG G(V,E): Consider a dominating Intutionistic fuzzy Graph $\mathrm{G}=(\mathrm{V}, \mathrm{E}, \mu$,

$\left.\gamma, \mu_{1}, \gamma_{1}\right)$ where $\mathrm{v}=\left\{\mathrm{v}_{1}, \mathrm{v}_{2}, \mathrm{v}_{3}, \mathrm{v}_{4}\right\}$ and

and $\gamma_{1}$ are given by $\mu: V \rightarrow[0,1]$ and $\gamma V \rightarrow[0,1]$ where

$\mu_{1}\left(v_{1}\right)=\max \left[\mu\left(v_{1} v_{2}\right), \mu\left(v_{1} u_{1}\right), \mu\left(v_{1} u_{2}\right), \mu\left(v_{1} v_{4}\right)\right]$ $=\max [0.1,0.03,0.11,0.05]=0.11$.

$\mu_{1}\left(v_{2}\right)=\max \left[\mu\left(v_{2} u_{2}\right), \mu\left(v_{2} u_{1}\right), \mu\left(v_{2} v_{3}\right), \mu\left(v_{2} v_{1}\right)\right]$

$=\max [0.45,0.15,0.54,0.1]=0.54$.

$\mu_{1}\left(v_{3}\right)=\max \left[\mu\left(v_{3} u_{1}\right), \mu\left(v_{3} u_{2}\right), \mu\left(v_{3} v_{2}\right), \mu\left(v_{3} v_{4}\right)\right]$

$=\max [0.13,0.42,0.54,0.25]=0.54$.

$\mu_{1}\left(v_{4}\right)=\max \left[\mu\left(v_{4} v_{1}\right), \mu\left(v_{4} u_{2}\right), \mu\left(v_{4} v_{3}\right), \mu\left(v_{4} u_{1}\right)\right]$

$=\max [0.05,0.20,0.25,0.08]=0.25$. $\mu\left(u_{1} v_{2}\right) \leq \mu_{1}\left(u_{1}\right) \wedge \mu_{1}\left(v_{2}\right)$

$0.15 \leq 0.15 \wedge 0.54$

$\gamma\left(u_{1} v_{2}\right) \leq \gamma_{1}\left(u_{1}\right) \wedge \gamma_{1}\left(v_{2}\right)$

$0.05 \leq 0.05 \wedge 0.01$

Here $\mathrm{u}_{2}$ dominates $\mathrm{v}_{2}$ because

$\mu\left(u_{2} v_{2}\right) \leq \mu_{1}\left(u_{2}\right) \wedge \mu_{1}\left(v_{2}\right)$

$0.45 \leq 0.45 \wedge 0.54$

$\gamma\left(u_{2} v_{2}\right) \leq \gamma_{1}\left(u_{2}\right) \wedge \gamma_{1}\left(v_{2}\right)$

$0.01 \leq 0.01 \wedge 0.01$

Here $\mathrm{V}=\left\{\mathrm{v}_{1}, \mathrm{v}_{2}, \mathrm{v}_{3}, \mathrm{v}_{4}, \mathrm{u}_{1}, \mathrm{u}_{2}\right\}$ and $\mathrm{D}=\left\{\mathrm{v}_{1}, \mathrm{v}_{2}, \mathrm{v}_{3}, \mathrm{u}_{1}, \mathrm{u}_{2}\right\} \quad \mathrm{V}-$ $\mathrm{D}=\left\{\mathrm{v}_{4}\right\}$

$|\mathrm{D}|=5=$ sum of dominating elements

$\mu_{1}\left(u_{1}\right)=\max \left[\mu\left(u_{1} u_{2}\right), \mu\left(u_{1} v_{2}\right), \mu\left(u_{1} v_{1}\right), \mu\left(u_{1} v_{3}\right), \mu\left(u_{1} v_{4}\right)\right]^{(\mathrm{G})=}$

$=\max [0.12,0.15,0.03,0.13,0.08]=0.15$.

$(0,0)$

$(0.05,0.2) \quad(0.03,0.45)$

$(0.11,0.2)$

$\mu_{1}\left(u_{2}\right)=\max \left[\mu\left(u_{2} v_{1}\right), \mu\left(u_{2} v_{2}\right), \mu\left(u_{2} v_{4}\right), \mu\left(u_{2} v_{3}\right), \mu\left(u_{2} v\right)\right]$ $=\max [0.11,0.45,0.20,0.42,0.12]=0.45$

$\gamma_{1}\left(v_{1}\right)=\min \left[\gamma\left(v_{1} v_{2}\right), \gamma\left(v_{1} u_{1}\right), \gamma\left(v_{1} u_{2}\right), \gamma\left(v_{1} v_{4}\right)\right]$

$=\min [0.05,0.45,0.2,0.2]=0.05$.

$\gamma_{1}\left(v_{2}\right)=\min \left[\gamma\left(v_{2} u_{2}\right), \gamma\left(v_{2} u_{1}\right), \gamma\left(v_{2} v_{3}\right), \gamma\left(v_{2} v_{1}\right)\right]$

$=\min [0.01,0.05,0.01,0.05]=0.01$.

$\gamma_{1}\left(v_{3}\right)=\min \left[\gamma\left(v_{3} u_{1}\right), \gamma\left(v_{3} u_{2}\right), \gamma\left(v_{3} v_{2}\right), \gamma\left(v_{3} v_{4}\right)\right]$

$=\min [0.15,0.08,0.01,0.1]=0.01$.

$\gamma_{1}\left(v_{4}\right)=\min \left[\gamma\left(v_{4} v_{1}\right), \gamma\left(v_{4} u_{2}\right), \gamma\left(v_{4} v_{3}\right), \gamma\left(v_{4} u_{1}\right)\right]$

$=\min [0.2,0.20,0.1,0.35]=0.1$.

$\gamma_{1}\left(u_{1}\right)=\min \left[\gamma\left(u_{1} u_{2}\right), \gamma\left(u_{1} v_{2}\right), \gamma\left(u_{1} v_{1}\right), \gamma\left(u_{1} v_{3}\right), \gamma\left(u_{1} v_{4}\right)\right]$

$=\min [0.28,0.05,0.45,0.15,0.35]=0.05$.

$\gamma_{1}\left(u_{2}\right)=\min \left[\gamma\left(u_{2} v_{1}\right), \gamma\left(u_{2} v_{2}\right), \gamma\left(u_{2} v_{4}\right), \gamma\left(u_{2} v_{3}\right), \gamma\left(u_{2} u_{1}\right)\right]^{\gamma_{D}}(G)=$

$=\min [0.2,0.01,0.20,0.08,0.28]=0.01$.

Here $v_{1}$ dominates $v_{2}$ because

$\mu\left(v_{1} v_{2}\right) \leq \mu_{1}\left(v_{1}\right) \wedge \mu_{1}\left(v_{2}\right)$

$0.1 \leq 0.11 \wedge 0.54$

$\gamma\left(v_{1} v_{2}\right) \leq \gamma_{1}\left(v_{1}\right) \wedge \gamma_{1}\left(v_{2}\right)$

$0.05 \leq 0.05 \wedge 0.01$

Here $v_{2}$ dominates $v_{3}$ because

$\mu\left(v_{2}, v_{3}\right) \leq \mu \mu_{1}\left(v_{2}\right) \wedge \mu_{1}\left(v_{3}\right)$

$\gamma\left(v_{2} v_{3}\right) \leq \gamma_{1}\left(v_{2}\right) \wedge \gamma_{1}\left(v_{3}\right)$

$0.54 \leq 0.54 \wedge 0.54$

$0.01 \leq 0.01 \wedge 0.01$

Here $v_{3}$ dominates $v_{4}$ because

$\mu\left(v_{3} v_{4}\right) \leq \mu_{1}\left(v_{3}\right) \wedge \mu_{1}\left(v_{4}\right)$

$0.25 \leq 0.54 \wedge 0.25$

$\gamma\left(v_{3} v_{4}\right) \leq \gamma_{1}\left(v_{3}\right) \wedge \gamma_{1}\left(v_{4}\right)$

$0.1 \leq 0.01 \wedge 0.1$

Here $\mathrm{u}_{1}$ dominates $\mathrm{v}_{2}$ because

$\left[\begin{array}{cccccc}\text { v } \\ \hline(0,0) & (0.54,0.01) & (1,1) & (0.25,0.1) & (0.13,0.15) & (0.42,0.08) \\ (0.05,0.2) & (0,0) & (0.25,0.1) & (0,0) & (0.08,0.35) & (0.20,0.20) \\ (0.03,0.45) & (0.15,0.05) & (0.13,0.15) & (0.08,0.35) & (1,1) & (0.12,0.28) \\ (0.11,0.2) & (0.45,0.01) & (0.42,0.08) & (0.20,0.20) & (0.12,0.28) & (1,1)\end{array}\right]$

$\begin{aligned} \mu_{D}(G) & =\left[\begin{array}{cccccc}1 & 0.1 & 0 & 0.05 & 0.03 & 0.11 \\ 0.1 & 1 & 0.54 & 0 & 0.15 & 0.45 \\ 0 & 0.54 & 1 & 0.25 & 0.13 & 0.42 \\ 0.05 & 0 & 0.25 & 0 & 0.08 & 0.02 \\ 0.03 & 0.15 & 0.13 & 0.08 & 1 & 0.12 \\ 0.11 & 0.45 & 0.42 & 0.02 & 0.12 & 1\end{array}\right] \\ \gamma_{D}(G) & =\left[\begin{array}{cccccc}1 & 0.05 & 0 & 0.2 & 0.45 & 0.2 \\ 0.05 & 1 & 0.11 & 0 & 0.05 & 0.01 \\ 0 & 0.11 & 1 & 0.1 & 0.15 & 0.08 \\ 0.2 & 0 & 0.1 & 0 & 0.35 & 0.02 \\ 0.45 & 0.05 & 0.15 & 0.35 & 1 & 0.28 \\ 0.2 & 0.01 & 0.08 & 0.02 & 0.28 & 1\end{array}\right]\end{aligned}$

Eigen values of $\mu_{\mathrm{D}}(\mathrm{G})=[-$

$0.1025,0.5084,0.5989,0.9467,1.0014,2.0471]=5.205$.

Eigen values of $\gamma_{\mathrm{D}}(\mathrm{G})=[-$

$0.1263,0.5294,0.8161,0.9902,1.0090,1.7816]=5.2526$.

Dominating Energy of Join of IFG $\bar{G}=(\bar{V}, \bar{E})$ is =

$\left[\sum_{\lambda_{i} \in X}\left|\bar{\lambda}_{i}\right|, \sum_{\delta_{i} \in Y}\left|\bar{\delta}_{i}\right|\right]=(5.205,5.2526)$

Eigen values of $\gamma_{D}\left(G_{1} \cup G_{2}\right)=\{-0.0731$,-

$0.0478,0.9663,1.0033,1.0731,1.0782\}=4.2418$.

\section{Conclusion}

In this paper, we have defined the dominating energy in different operations of intuitionistic fuzzy graphs and examined the domi- 
nating energy on complement of an Intuitionistic fuzzy graph , union and join of the two intuitionistic fuzzy graphs.

\section{References}

[1] Anjali.N, Sunil Mathew, Energy of a fuzzy graph of Annals of Fuzzy Mathematics and Informatics, (2013).

[2] Atanassov.K, Intuitionistic fuzzy sets, Fuzzy sets and systems, 20 , 87-96, (1986).

[3] Atanassov.K, Intuitionistic Fuzzy sets : Theory and Applications, Springer-Verlag, Heidelberg, (1999).

[4] Cockayne.E.J and Hedetnieme, S.T, Towards a Theory of Domination in Graphs, Networks 7, 247-261, (1977).

[5] De Jaenisch,C.F. Applications de 1' Analyze Mathematique an Jen des Echecs, (1862).

[6] Deepa.G, Praba.B,Chandrasekaran.V.M,Virus Spread in an Intuitionistic Fuzzy Network of International Journal of Applied Engineering Research,Vol.9, No.16, pp.5507- 5515(2014).

[7] Deepa.G, Praba.B, Chandrasekaran.V.M, Max-Min Intuitionistic Fuzzy Matrix of an Intuitionistic Fuzzy Graph,International Journal of Pure and Applied Mathematics, Vol.98, No.3, 375-387,(2015).

[8] Gutman,I. The Energy of a Graph, Ber. Math - Statist. Sekt. Forschungsz. Graz, 103, 1-22, (1978).

[9] Gutman.I, The energy of a graph: old and new results, in Algebraic Combinatorics and Applications, A. Betten, A. Kohner, R. Laue, and A.Wassermann, eds., Springer, Berlin, 196-211 (2001).

[10] Nagoorgani.A and Chandrasekaran.V.T, Domination in Fuzzy Graph, Advances in Fuzzy Sets and systems, 1(1),17-26 (2006).

[11] Parvathi R, Karumbigai M G. Intutionistic fuzzy graphs. Computational Intelligence, Theory and Applications. 139- 150.

[12] Parvathi $\mathrm{R}$ and G. Thamizhendhi, Domination in intuitionistic fuzzy graphs, Fourteenth Int. Conf. on IFSs, Sofia, 15-16 May 2010 ,NIFS Vol. 16 (2010), 2, 39-49

[13] Praba.B, Chandrasekaran.V.M., Deepa.G, Energy of an Intuitionistic Fuzzy Graph of Italian journal of pure and applied mathematics-N.32,431-444, (2014).

[14] Rosenfeld.A, Fuzzy Graphs, in Zadeh.L.A, Fu. K.cS, Tanaka.K and Shimura.M (eds.), Fuzzy Sets and their Applications to Cognitive and Decision Process, Academic Press, New York, 75-95 (1975).

[15] Somasundaram.A and Somasundaram.S, Domination in Fuzzy Graphs - I, Elsevier Science, 19, 787-791 (1998).

[16] Sharief Basha.S and E. Kartheek, Laplacian Energy of an Intuitionistic Fuzzy Graph, Indian Journal of Science and Technology, ,Vol 8(33), 1-7, (Dec2015).

[17] Vinoth Kumar.N, Geetha Ramani.G, Some Domination Parameters of the Intuitionistic Fuzzy graph and its Properties, International Journal of Mathematics and Scientific .Compting, Vol.1, No.1, (2011).

[18] Zadeh.L.A., Fuzzy sets , Information and Control, 1965; 8: 338353. 